\title{
Graphical Comparison of Coronary Arterial Culprit Lesions in Acute Myocardial Infarction and Unstable Angina Pectoris
}

\author{
Noriyo Namiki, Takashi UchiYama, Yoshikazu Nagai and Akira YamashinA*
}

\begin{abstract}
Objective The culprit lesion morphology at acute myocardial infarction (AMI) and unstable angina pectoris (UAP) was investigated by observing the responsible vessels through intravascular ultrasound (IVUS) during the acute stage. Methods As the subjects of study, 54 lesions of 54 ACS patients (26 in AMI patients, 28 in UAP patients) were enrolled prospectively from June 1994 to June 1998. The appearance of plaque in the lesion, the distal and proximal sites, extent of calcification, eccentricity, remodeling and shrinkage were observed through IVUS before the intervention. Results At lesion and distal site, significantly more soft plaques were observed in AMI than UAP. As to the extent of calcification in the former, mild calcification was noted significantly more in distal site as well as a tendency of more mild calcification in the lesion and proximal site. Conclusion These results suggested that the condition of responsible coronary artery is involved in the onset mechanism of AMI and UAP.

(Internal Medicine 38: 849-855, 1999)
\end{abstract}

Key words: intravascular ultrasound, acute coronary syndromes

\section{Introduction}

Acute myocardial infarction (AMI) and unstable angina pectoris (UAP) have recently come to be called acute coronary syndromes (ACS), because they have been found to demonstrate the same pathology in which occlusion or stenosis of the coronary artery lumen is caused by the rupture of fragile plaque and the subsequently formed thrombus $(1-4)$.

There are many reports which suggest the pathological involvement of thrombus in the onset mechanism of ACS (3-9). Clinical investigations using angioscopes also have confirmed the presence of thrombus obstructing the lumen of the coronary artery as the culprit lesion morphology of AMI (10-12). Using intravascular ultrasound (IVUS), Torre et al (13) and Rasheed et al $(14,15)$ reported that more soft plaque and calcification was observed in UAP than in stable angina pectoris. These may be explained by the differential mechanisms of occlusive plaque formation in these 2 syndromes. However, there are few reports, comparing AMI with UAP in relation to the morphology and appearance of plaque. In the present study, we investigated the difference in the morphology and appearance of plaque between AMI and UAP covering the culprit lesion morphology of ACS using IVUS.

\section{Subjects}

A total 54 ACS patients (54 lesions) were enrolled prospectively from June 1994 through June 1998. All cases in which intervention was performed within 48 hours after onset and in whom IVUS examination of the responsible coronary artery was performed prior to intervention were considered. There were 40 males and 14 females. Their mean age was $63.5 \pm 9.4$ years old. These 54 ACS patients were classified as 26 AMI patients (26 lesions) and 28 UAP patients (28 lesions).

Patients satisfying the following 3 criteria were given a diagnosis of AMI: 1) Chest pain persisting for more than 20 minutes; 2) ST increase of more than $0.1 \mathrm{mV}$ in at least more than 2 adjacent leads in ECG; 3 ) Increase in creatine phosphokinase (CPK) to a level double the normal value.

In contrast, cases were considered to have UAP if coronary reconstruction was judged necessary within 48 hours of onset, and if they had a severity of I or III (I; new onset severe or accelerated angina, No rest pain, III; Angina at rest within 48 hours), clinical symptom of B (B; primary unstable angina) and a treatment status of 2 or 3 (2; appropriate therapy for chronic stable angina, 3 ; maximally tolerated doses of all three categories of antiischemic drugs, including intravenous nitroglycerin) according to Braunwald's (16) classification.

From the Department of Cardiology Hachioji Medical Center of Tokyo Medical University, Tokyo and *the Second Department of Internal Medicine Tokyo Medical University, Tokyo

Received for publication October 7, 1998; Accepted for publication May 29, 1999

Reprint requests should be addressed to Dr. Noriyo Namiki, the Department of Cardiology Hachioji Medical Center of Tokyo Medical University, 1163, Tatemachi, Hachioji, Tokyo 193-0014 


\section{NAMIKI et al}

\section{Methods}

\section{Procedural technique}

For coronary angiography, heparin (3,000 units) was administered, after which the right and left coronary arteries were imaged by the femoral artery approach using a $6 \mathrm{Fr}$ catheter to identify the responsible coronary artery. Subsequently, an $8 \mathrm{Fr}$ guide catheter was inserted into the responsible coronary artery followed by additional administration of 7,000 units of heparin. Then a 0.014-inch guide wire (Bare wire) was passed through the lesion. Using this as the guide, before intervention, a 3.5Fr IVUS catheter (Endosonic Co., Ltd., Calfolnia 20 $\mathrm{MHz}$ ) was inserted and the whole responsible coronary artery was observed by pulling the IVUS catheter from the peripheral side of the distal site of the lesion as much as possible using the automatic pullback method or by manual movement. Quantitative coronary angiography was measured using an automated computer-assisted edge detection algorithm with magnification, calibrated from the guiding catheter diameter using a CARDIO 500 (Kontron Co., Ltd., müchen). Ultrasound images were recorded on a video tape for later review and ultrasound image analysis. Then, quantitative coronary ultrasound was done by the trace method using a CARDIO 500. Quantitative coronary angiography and quantitative coronary ultrasound were performed by separate units in the angiographic laboratory without the knowledge of the other results. Those in the AMI group from which peripheral site information was not captured by angiography after passing the Bare wire through the lesion were excluded. Full informed consent for the IVUS procedure was obtained from all of the patients. The following items were compared between the AMI group and UAP group.

\section{Ultrasound image analysis}

The appearance of plaque and extent of calcification in the lesion and sites within $10 \mathrm{~mm}$ distal and proximal from lesions were examined. When a side branch was found within $10 \mathrm{~mm}$ of a lesion, we observed the plaque within the side branch.

The appearance of plaque was classified into 3 types namely (17): soft plaque, mixed plaque and hard plaque. The plaque was classified as soft plaque if there was no calcification, the brightness was less than that of the adventitia and if a uniform echo accounted for up to $80 \%$ of plaque. The plaque was classified as hard plaque if there was no calcification and a uniform echo with brightness similar to or higher than that of adventitia accounted for more than $80 \%$ of the plaque. Plaque showing the mixed presence of calcified lesions and fibrous plaque was classified as mixed plaque. Furthermore, lesions showing soft plaque were termed "plaque I" while the mixed plaque or hard plaque types were termed "plaque II".

\section{Eccentricity, remodeling and shrinkage}

Concerning the extent of calcification, lesions showing no calcification were graded as 0 , those with calcification extending less than $90^{\circ}$ around the circumference of the vessel as $1+$, those with calcification extending for $90^{\circ}$ to $180^{\circ}$ around the circumference as $2+$, and more than $180^{\circ}$ as $3+$. Lesions assessed as 0 or $1+$ were classified as mild calcification and those assessed as $2+$ or $3+$, as advanced calcification (18).

Lesions assessed as less than 0.5 on the eccentric index were regarded as eccentric.

Eccentric index $=$ the thinnest wall thickness of plaque/contralateral wall thickness

When the vascular area of the lesion was larger than the vascular area proximal and distal to the lesion this was regarded as remodeling. However it was classified as shrinkage when the vascular area of the lesion was smaller than the proximal and distal vascular areas.

\section{Quantitative coronary ultrasound}

Minimal lumen diameter (MLD)

Lumen area (LA)

Total vessel area (TVA)

$\%$ Plaque area $(\% \mathrm{PA})=($ TVA-LA $) / \mathrm{TVA} \times 100$

\section{Quantitative coronary angiography}

Reference vessel (mean of proximal and distal to the lesion site) and lesion site minimal lumen diameters were used to derive percent diameter stenosis (\% stenosis).

\section{Statistical analysis}

Data are expressed as categorical variables and as means \pm one standard deviation for continuous variables. Chi-square analysis was used to assess the difference in categorical variables and the unpaired Student's t-test to assess differences in continuous variables. Values of $p<0.05$ were considered to be significant.

\section{Results}

\section{Patient background}

No significant difference was observed between the AMI group and UAP group for any of the background factors including age, male/female ratio, responsible vessel, and coronary risk factors (presence or absence of smoking, hypertension, hyperlipidemia, diabetes mellitus) (Table 1).

\section{Quantitative coronary angiography and quantitative coronary ultrasound}

Table 2 shows the \%stenosis, MLD, LA, TVA and \%PA in the AMI and UAP groups. \%stenosis was higher in the AMI group than in the UAP group $(94.06 \pm 9.50 \%$ vs $86.97 \pm 8.46 \%$, $\mathrm{p}<0.05$ ) and MLD of the lesion was significantly lower in the AMI group than in the UAP group $(1.76 \pm 0.29 \mathrm{~mm}$ vs $1.95 \pm 0.49$ $\mathrm{mm}, \mathrm{p}<0.05)$. No significant difference was noted in the other data (Table 2).

\section{Morphology of plaque}

Compared with the UAP group, there were more plaques classified as plaque I in distal sites area and lesion in the AMI group, demonstrating a significant difference $(p<0.05)$. There was no significant difference in the plaque in the proximar site 


\section{Graphical Comparison in AMI and UAP}

between the groups. There was a tendency for more plaques to be assessed as plaque II in distal sites, lesion and proximal sites in the UAP group (Fig. 1).

\section{Extent of calcification}

Mild calcification was significant by more frequent in the AMI group than in the UAP group $(\mathrm{p}<0.05)$. A tendency torward milder calcification was noted in all sites in the AMI group (Fig. 2)

Table 1. Patient Characteristics

\begin{tabular}{lccc}
\hline & AMI & UAP & p-value \\
\hline $\begin{array}{l}\text { No. of cases } \\
\text { Age (yr) }\end{array}$ & 26 & 28 & N.S. \\
Gender & $62.7 \pm 12.1$ & $63.0 \pm 7.7$ & N.S. \\
$\quad$ Male & 17 & 23 & N.S. \\
& 9 & 5 & N.S. \\
Vessel & & & \\
LAD & 13 & 19 & N.S. \\
Lcx & 5 & 2 & N.S. \\
RCA & 8 & 7 & N.S. \\
Risk Factor & & & \\
Smoking & 9 & 8 & N.S. \\
HT & 10 & 13 & N.S. \\
HL & 15 & 16 & N.S. \\
DM & 11 & 8 & N.S. \\
\hline
\end{tabular}

N.S: not significant, AMI: acute myocardial infarction, UAP: unstable anginal pectoris, LAD: left anterior descending artery, Lcx: left circumflex artery, RCA: right coronary artery, HT: hypertension, HL: hyperlipidemia, DM: diabetes mellitus.

\section{Extent of eccentricity}

The eccentricity score was $65.4 \%$ in the AMI group and $75.0 \%$ in the UAP group, but there was no statistically significant difference (Table 3 ).

\section{Presence or absence of remodelling and shrinkage}

In comparison with the AMI group, more remodeling and less shrinkage were noted in the UAP group (15.4\% vs $42.9 \%$, $\mathrm{p}<0.05$; and $38.5 \%$ vs $10.1 \%$, $\mathrm{p}<0.05$, respectively) (Table 3 ).
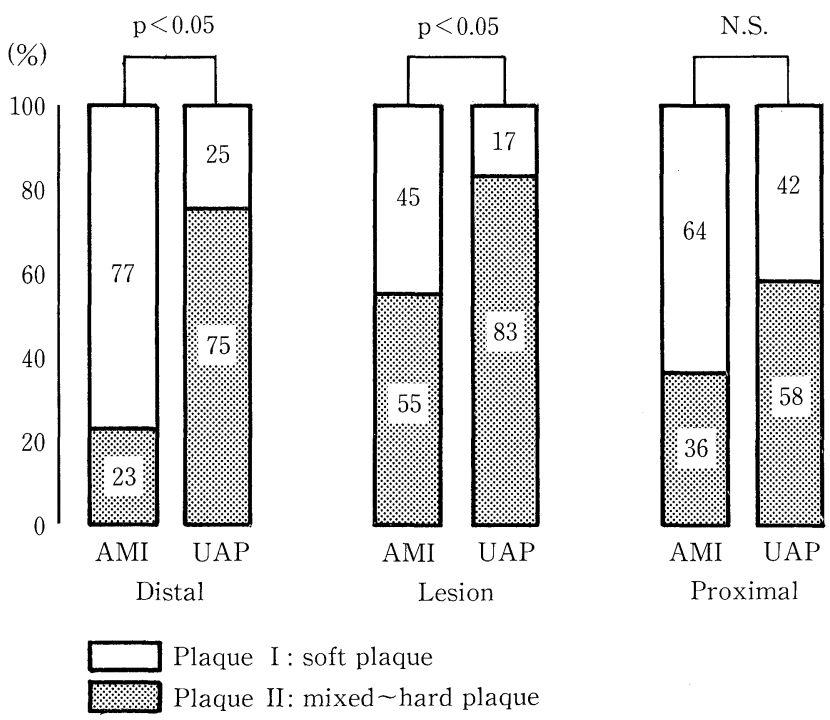

Figure 1. Characteristics of plaque. N.S: not significant, AMI: acute myocardial infarction, UAP: unstable angina pectoris.

Table 2. Quantitative Coronary Angiography and Quantitative Coronary Ultrasound

\begin{tabular}{llccc}
\hline & & $\begin{array}{c}\text { AMI } \\
(\mathrm{n}=26)\end{array}$ & $\begin{array}{c}\text { UAP } \\
(\mathrm{n}=28)\end{array}$ & p value \\
\hline \% stenosis $(\%)$ & pre & $94.06 \pm 9.50$ & $86.97 \pm 8.46$ & $<0.05$ \\
MLD $(\mathrm{mm})$ & distal & $2.73 \pm 0.46$ & $2.96 \pm 0.71$ & N.S. \\
& lesion & $1.76 \pm 0.29$ & $1.95 \pm 0.49$ & $<0.05$ \\
& proximal & $2.98 \pm 0.60$ & $3.48 \pm 0.96$ & N.S. \\
LA $\left(\mathrm{mm}^{2}\right)$ & distal & $7.29 \pm 2.38$ & $8.68 \pm 4.00$ & N.S. \\
& lesion & $3.20 \pm 1.10$ & $3.89 \pm 1.78$ & N.S. \\
& proximal & $9.11 \pm 3.47$ & $10.81 \pm 4.19$ & N.S. \\
TVA $\left(\mathrm{mm}^{2}\right)$ & distal & $13.91 \pm 6.37$ & $15.38 \pm 5.68$ & N.S. \\
& lesion & $15.79 \pm 7.22$ & $14.67 \pm 3.47$ & N.S. \\
& proximal & $17.71 \pm 6.35$ & $19.05 \pm 5.29$ & N.S. \\
& distal & $43.39 \pm 12.90$ & $44.83 \pm 12.12$ & N.S. \\
& lesion & $76.74 \pm 10.03$ & $72.07 \pm 14.97$ & N.S. \\
& proximal & $46.53 \pm 14.65$ & $45.24 \pm 15.60$ & N.S. \\
\hline
\end{tabular}

MLD: minimal lumen diameter, LA: lumen area, TVA: total vessel area, \%PA: \%plaque area. 


\section{NAMIKI et al}

\section{Typical cases are shown below}

Typical AMI case

A 44-year-old male was admitted for the treatment of acute anteroseptal myocardial infarction and urgent coronary angiog-
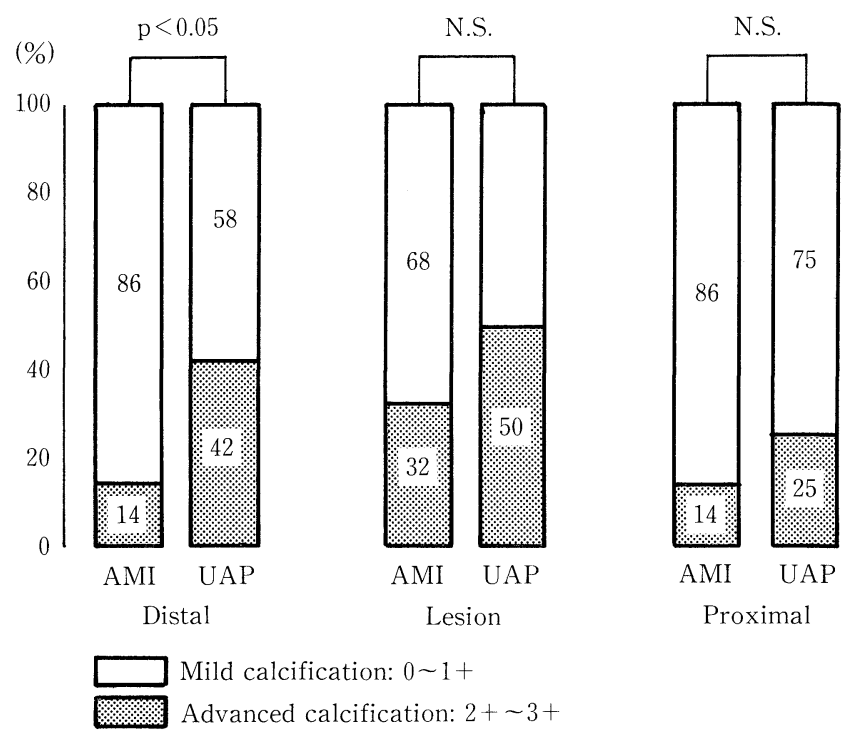

Figure 2. Extent of calcification. raphy was immediately done. The proximal LAD showed $84 \%$ lumen narrowing. IVUS detected a crescent echo lucent zone (*) lacking internal echo from the proximal region to the lesion. Furthermore, a thin fibrous cap was also noted in the proximal site of the lesion and along with it, there was a tear protruding into the lumen in the direction of 3 to 5 o'clock ( $\downarrow$ ). This was considered to be a finding indicative of rupture of plaque. There was a soft plaque assumed to be eccentric and movable thrombus-like echo in the lesion while only soft plaque was noted in the distal site (Fig. 3)

\section{Typical UAP case}

A 53-year-old male was admitted for the treatment of fresh onset of unstable angina. It was difficult to control the attack

Table 3. Characteristics of Plaque

\begin{tabular}{lccc}
\hline & $\begin{array}{c}\text { AMI } \\
(\mathrm{n}=26)\end{array}$ & $\begin{array}{c}\text { UAP } \\
(\mathrm{n}=28)\end{array}$ & $\mathrm{p}$ value \\
\hline Eccentric & $65.4 \%(17)$ & $75.0 \%(21)$ & N.S. \\
Remodeling & $15.4 \%(4)$ & $42.9 \%(12)$ & $\mathrm{p}<0.05$ \\
Shrinkage & $38.5 \%(10)$ & $10.1 \%(3)$ & $\mathrm{p}<0.05$ \\
\hline
\end{tabular}

N.S: not significant, AMI: acute myocardial infarction, UAP: unstable angina pectoris.

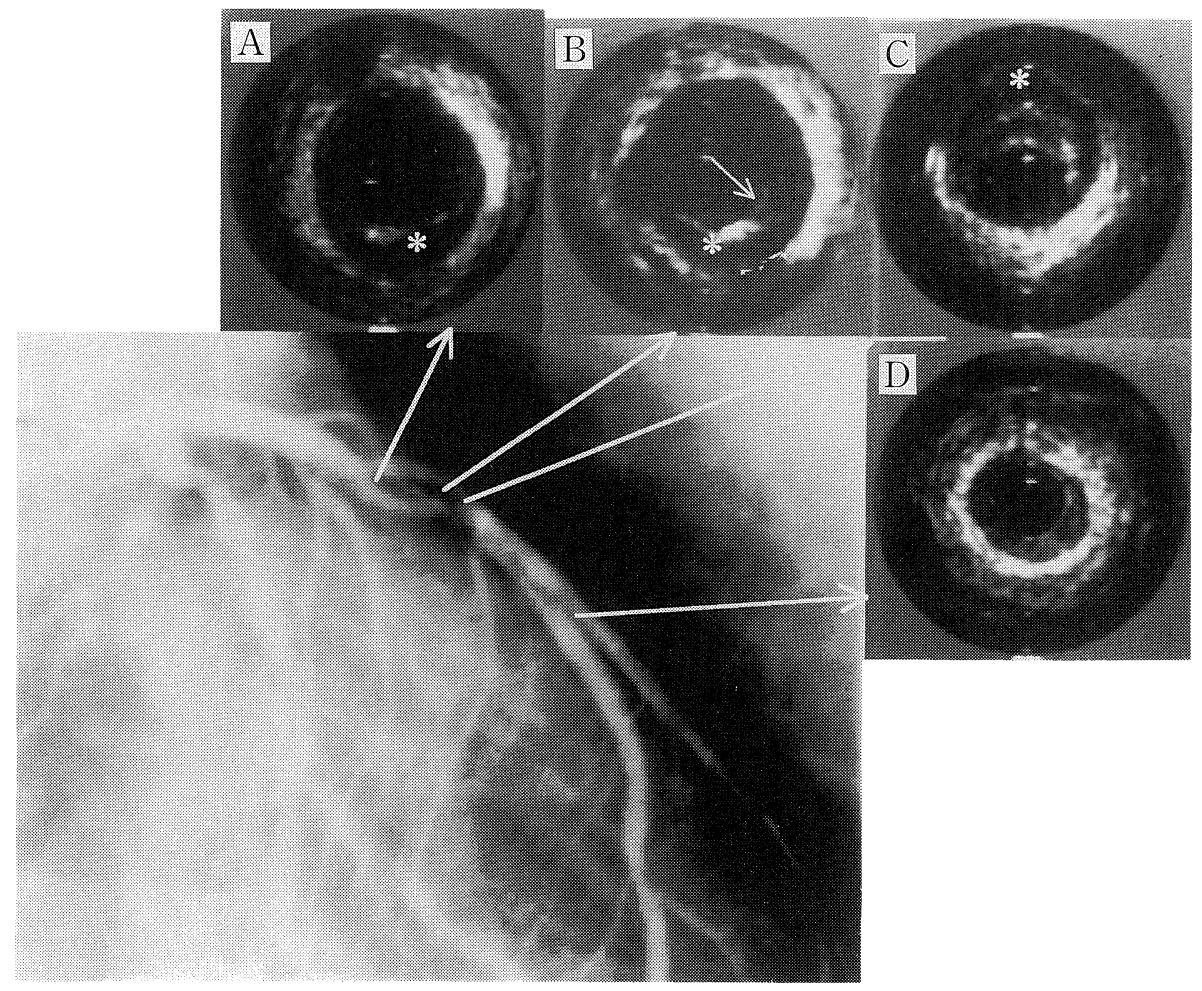

Figure 3. Case 1. A, B; proximal to the lesion, C; lesion, D; distal to the lesion. IVUS detected a crescent echo lucent zone $(*)$ lacking internal echo proximal to the lesion. Furthermore, a thin fibrous cap was also noted in the proximal site of lesion and along with it, there was a tear protruding into the lumen in the direction of 3 to 5 o'clock ( $\downarrow$ ). 


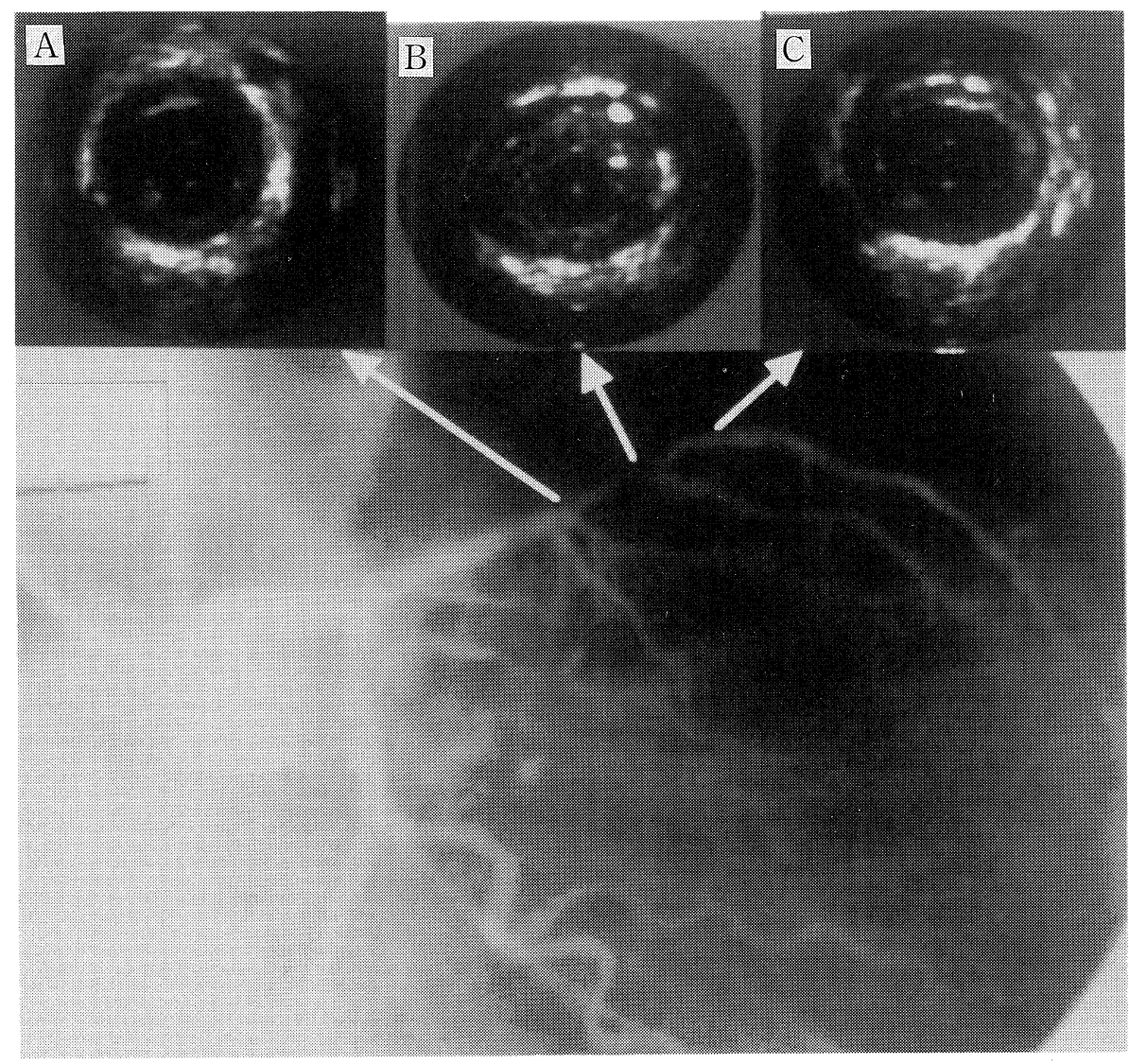

Figure 4. Case 2. A; proximal to the lesion, B; lesion, C; distal to the lesion. IVUS detected concentric soft plaque in the proximal site of lesion. Mixed plaque demonstrating partial calcification filling the lumen in the lesion. Mixed plaque with the calcification of +2 was observed in the distal site. Remodeling was noted in this case.

even after admission and urgent coronary angiography was conducted on the second day of admission. The proximal LAD showed $78 \%$ lumen narrowing and a concentric soft plaque was detected on IVUS in the proximal site of lesion. Mixed plaque partly demonstrating calcification filled the lumen in the lesion. Mixed plaque with calcification of +2 was observed in the distal site. Remodeling was noted in this case (Fig. 4).

\section{Discussion}

The culprit lesion morphology of AMI was considered to be different from that of UAP. However, DeWood et al (8) reported in 1980 that complete occlusion by thrombus which wasassumed to be caused by the rupture of plaque was noted in $87 \%$ of their autopsied AMI cases. Furthermore, they reported that unstable plaque accompanying thrombus was observed in deteriorating angina pectoris cases and smooth plaque was observed in stable angina cases. A recent pathological investigation by Fuster et al $(4,9)$ in 1992 generalized AMI and UAP as ACS because the rupture of plaque and the associated thrombus were common characteristics observed in the culprit lesion plaque morphology of AMI and UAP. The onset mecha- nism of ACS is described as follows: the progress of pultaceous sclerosis promotes the growth of smooth muscle cells and infiltration of foam cells as well as decomposition of the extracellular matrix caused by monocytes, which makes plaque fragile and susceptible to rupture $(19,20)$.

The plaque consequently ruptures due to some external stress (such as a change in pulse pressure, coronary contraction) to the fragile fibrous cap. The rupture of plaque promotes platelet aggregation and coagulation at the site in question, forming thrombus. The AMI is assumed to be caused by advanced stenosis of the coronary lumen by thrombus formation in addition to UAP occlusion. In classifying thrombus by the tone captured on angioscopy, there are red thrombi (whole thrombus appears red) and white thrombi (the whole thrombus appears white) as well as mixed thrombi (mixed red and white). There is a report that more white thrombi and non-occlusive thrombi are noted in UAP (10). However, the in vivo onset mechanism of ACS has not yet been clarified. Thus, the difference in the thrombi formation between AMI and UAP was investigated by observing the responsible coronary artery through IVUS. 


\section{Quantitative coronary analysis}

No significant difference between the AMI and UAP groups in LA, TVA or \%PA was observed at any site, indicating that these parameters were not involved in the onset mechanism of AMI and UAP. However, there was a significant difference in $\%$ stenosis and MLD of lesions. By taking the complete occlusion in AMI as $100 \%$, and then the measurement of \%stenosis, this contributed to a significantly higher value in AMI when the two groups were compared. When the mural thrombus is mobile in the lumen, the shadow is sometimes captured on IVUS as an ambiguous echo moving in correspondence with the cardiac rhythm (21). However, as the echo brightness of the thrombus and that of plaque in the lumen may change depending on the time of formation and component (22). it is difficult to differentiate the thrombus from plaque. In other words, it is possible that the thrombotic portion was included in the determination of MLD, which resulted in an error in MLA. However, Horie et al (23) reported that pathological investigation of AMI indicated that stenosis of more than $75 \%$ in the coronary lumen was seen in $69.1 \%$ and that stenosis of less than $50 \%$ accounted for $8.7 \%$, indicating that the occlusion by thrombus is more likely to occur in patients with advanced lesions in the coronary lumen.

\section{Appearance and calcification of plaque}

In general, it is considered that there are more soft plaques in ACS in comparison with stable angina. According to the report by Torre et al (13), soft plaque was observed in $75 \%$ of unstable angina and in $25 \%$ of stable angina. The present results indicated more soft plaques distal to the lesion and in the lesion in AMI group while mixed plaques and hard plaques tended to be more prominant at all sites in the UAP group. It is assumed that the softer the plaque, the more unstable the plaque, resulting in larger thrombus formation at the time of rupture. Calcification is reportedly less in ACS. The present results also indicated less advanced calcification in the lesion, and in distal and proximal sites in AMI and UAP groups.

Both groups demonstrated a high rate of mild calcification. Significantly milder calcification was observed in the sites distal to the lesion of AMI patients. Based on the above results, it is considered that there is a tendency towards less calcification of responsible coronary artery and more soft plaque in AMI in comparison with UAP, indicating that the extent of plaque rupture, and furthermore, the extent of thrombus formation, differs depending on the clinical pathology of ACS. In addition to coronary stenosis, coronary contraction and residual thrombus, the tissue substrate contributes to thrombus formation (9). The extent of calcification is a factor, which determines the stiffness of the fibrous cap, and its presence was experimentally shown to inhibit the rupture of plaque (24). It is therefore considered that thrombus formation by the rupture of plaque could be small in scale and could escape complete occlusion if there is more calcification and the plaque is hard. However, when ACS was classified into the fresh onset type and chronic deterioration type with long disease history, it was reported that the incidence of soft plaque was higher in the former (25).
It is therefore considered necessary to investigate the duration of symptoms and history of angina as well.

\section{Remodeling and shrinkage}

Glagov et al (26) in 1987 reported that the vascular lumen was maintained and the vessel itself is remodeled outward in the initial stage of atherosclerosis but that lumen stenosis occurs when the plaque occupies more than $40 \%$ of the total vascular area. On the other hand, the concept opposite to that reported by Glagov et al, that is, shrinkage of the vessel itself, has recently been observed in human coronary artery and femoral artery $(27,28)$. However, the mechanism of onset has yet to be clarified. We observed that TVA seemed to shrink in UAP (Table 1). But comparing each coronary artery, both remodeling and shrinkage were observed in AMI as well as in UAP but remodeling was especially remarkable in UAP while shrinkage was observed more often in AMI.

These results suggested that demonstrating remodeling, the lumen escaped occlusion by thrombus, which is generated after rupture of plaque in UAP, because the lumen was maintained to some extent. On the other hand, the lumen was occluded by thrombus produced after rupture of plaque in AMI, which demonstrated shrinkage, because the lumen became narrower.

UAP is this study corresponds to severity III or I by Braunwald's classification. A difference in the involvement of thrombus is conceivable between the groups I and III. It is therefore necessary to investigate this point by using angioscopy in the future.

\section{Conclusion}

When acute stage ACS was investigated by IVUS, calcification in the distal site of the lesion was more intense, demonstrating remodeling of the lesion in UAP patients while calcification in the distal site of lesion was small and shrinkage of the lesion was observed in AMI patients. Accordingly, it is considered more likely that a large quantity of thrombi is formed by the rhexis of plaque and that in AMI patients the lumen is susceptible to occlusion due to shrinkage.

\section{References}

1) Amborse JA, Winters SL, Arora RR, et al. Angiographic evalution of coronary artery morphology in unstable angina. J Am Coll Cardiol 7: 472-478, 1986.

2) Falk E. Morphologic features of unstable atherothrombotic plaques underlying acute coronary syndromes. Am J Cardiol 63: 114E-120E, 1989.

3) Fuster V, Badimon L, Badimon JJ, Chesebro JH. The pathogenesis of coronary artery disease and the acute coronary syndromes. $\mathrm{N}$ Engl $\mathrm{J}$ Med 326: 242-250, 1992.

4) Fuster V, Badimon L, Badimon JJ, Chesebro JH. The pathogenesis of coronary artery disease and the acute coronary syndromes. $\mathrm{N}$ Engl $\mathrm{J}$ Med 24: 326-318, 1992.

5) Friedman M. Pathogenesis of coronary thrombosis, intramural and intraluminal hemorrhage. Thrombosis and coronary heart disease, Adv Cardiol 4: 20-46, 1970.

6) Hamilton JD, Mowbray JH. The significance of intramural hemorrhages 


\section{Graphical Comparison in AMI and UAP}

in coronary atherosclerosis. Circulation 14: 486, 1956.

7) Sakurai I, Tosaka A, Yamada T, Matubuchi K. Pathogenesis of intraathromatous hematoma in coronary sclerosis. Arterial sclerosis 5: 259265, 1977.

8) DeWood MA, Spores J, Notske R, et al. Prevalence of total coronary occlusion during the early hours of transmural myocardial infarction. $\mathrm{N}$ Engl J Med 303: 879-902, 1980.

9) Fuster V. Lewis A. Conner Memorial Lectue. Mechanisms leading to myocardial infarction: Insights from studies of vascular biology. Circulation 90: 2126-2146, 1994.

10) Mizuno K, Satomura K, Miyamoto A, et al. Angioscopic evaluation of coronary syndromes. N Engl J Med 326: 287-291, 1992.

11) Escobar A, Ventura HO, Stapleton DD, et al. Native coronary atherosclerosis and cardiac allograft vasculopathy.: In vivo comparison by coronary angioscopy Circulation 90: I-489, 1994 (Abstract).

12) Hosokawa H, Suzuki T. Coronary angioscopy in patients with acute myocardial infraction undergoing direct balloon angioplasty. J Am Coll Cardiol 23: 346A, 1994 (Abstract).

13) Torre SR, Sharma SK, Almeida O, Goldman ME, Ambrose JA, Teichholz LE. Plaque morphology in acute coronary syndromes: New insights from intravascular ultrasound. J Am Coll Cardiol 21: 194A, 1993 (Abstract).

14) Rasheed Q, Nair RN, Sheehan HM, Hodgson JM. Coronary artery plaque morphology in stable angina and subsets of unstable angina: an in vivo intracoronary ultrasound study. Int J Card Imaging 27: 89-95, 1995.

15) Rasheed Q, Nair R, Sheehan H, Hodgson JM. Correlation of inracoronary ultrasound plaque characteristics in atherosclerotic coronary artery disease patient with clinical variables. Am J Cardiol 73: 753-758, 1994.

16) Braunwald E. Unstable angina: A classification. Circulation 80: 410-414, 1989.

17) Honye J, Moriuchi M. What can you measure by Intravascular ultrasound. Coronary imaging; intravascular ultrasound and angioscopy 53-58, 1996.

18) Honye J, Saito $S$, Moriuchi $M$, et al. Clinical application of intravascular ultrasound imaging. Circulation 58 Suppl 4: 1172-1176, 1994.
19) Falk E. Why do plaques rupture? Circulation 86 (Suppl III): III30-III42, 1992.

20) Stary HC, Chandler AB, Glagov S, et al, and A definition of initial, fatty streak, and intermediate lesions of atherosclerosis. A report from the Committee on Vascular Lesion of the Council on Arteriosclerosis, American Heart Association. Circulation 89: 2462-2478, 1994.

21) Pandian NG, Kreis A, Brockway B. Detection of intraarterial thrombus by intravascular high frequency two-dimensional ultrasound imaging in vitro and in vivo studies. Am J Cardiol 65: 1280-1283, 1990.

22) Mizushige $K$, Morita $H$, Senda S, Matsuo $H$. Evaluation of arterial wall histogical and physical characteristics using intravascular echography. Jpn Circ J 56: 565-571, 1992.

23) Horie T, Sekiguchi M, Hirosawa K. Coronary thrombosis in pathogenesis of acute myocardial infarction. Histopathological study of coronary arteries in 108 necropsied cases using serial section. Br Heart J 40: 153$161,1978$.

24) Lee RT, Grodzinsky AJ, Frank EH, Kamm RD, Schoen FJ. Structuredependent dynamic mechanical behavior of fibrous caps from human atherosclerotic plaques. Circulation 83: 1764-1770, 1991.

25) Moriuti M, Saitou S. Acute Coronary syndrome using intravascular echography. Acute Coronary Syndrome: 78-88: 1997.

26) Glagov S, Weisenberg E, Zarins CK, Stankunavicius R, Kolettis GJ. Compensatory enlargement of human atherosclerotic coronary arteries. $\mathrm{N} \mathrm{Engl}$ J Med 316: 1371-1375, 1987.

27) Nishioka T, Luo H, Eigler NL, Berglund H, Kim CJ, Siegel RJ. Contribution of inadequate compensatory enlargement to development of human coronary artery stenosis: an in vivo intravascular ultrasound study. J Am Coll Cardiol 27: 1517-1576, 1996.

28) Pasterkamp G, Wensing PWJ, Post MJ, Hillen B, Mali WPTN, Borst C. Paradoxical arterial wall shrinkage may contribute to luminal narrowing of human atherosclerotic femoral arteries. Circulation 91: 1444-1449, 1995. 